\title{
Avaliação da sustentabilidade da pecuária leiteira na Bacia Hidrográfica da Pedra Preta
}

\author{
Danilo Costa de-Almeida ${ }^{1 *}$, Adriana Cavalieri Sais ${ }^{2}$, Karla Beatriz Lopez Baldini ${ }^{3}$
}

\author{
Discente do Programa em Agroecologia e Desenvolvimento Rural, Universidade Federal de São Carlos \\ Docente do Departamento de Desenvolvimento Rural, Universidade Federal de São Carlos \\ Docente na Associação Educacional Dom Bosco \\ *Autor para correspondência: danilo.cost.almeida@gmail.com
}

Recebido em 05 de abril de 2018. Aceito em 14 de dezembro de 2019. Publicado em 27 de dezembro de 2019.

Resumo - A determinação do índice de sustentabilidade agroecológica em propriedades rurais é essencial para o direcionamento de ações de planejamento e execução de políticas públicas de assistência técnica e extensão rural, que promovam melhorias na qualidade de vida desta população. Neste trabalho foram analisados pelo método MESMIS um grupo de 25 indicadores de sustentabilidade ambiental, social e econômica de uma população de agricultores familiares residentes na Bacia Hidrográfica da Pedra Preta no Município de Resende RJ. Essa proposta teve como objetivo entender se há sustentabilidade no modelo de manejo das propriedades e apontar o índice de sustentabilidade para a comunidade e propriedades que dependem da agropecuária leiteira. Foram avaliadas 26 propriedades com aplicação dos indicadores, os quais abrangeram as dimensões socioeconômica e ambiental, onde o índice médio de sustentabilidade calculado foi de 67,8\%. De acordo com a classificação MESMIS, há fragilidades aparentes no tratamento de efluentes, no desconhecimento da agroecologia e na educação ambiental. Os produtores consultados se mostraram favoráveis a possíveis mudanças nos sistemas de manejo, mas observou-se que há carência de apoio técnico para a ação.

Palavras-chave: Agroecologia, diagnóstico, indicadores.

\section{Sustainability evaluation of dairy cattle in the Hydrographic Basin of Pedra Preta.}

Abstract - The determination of the agroecological sustainability index in rural properties is essential for the direction of planning actions and execution of public technical assistance and rural extension policies, which promote improvements in the quality of life of these populations. In this work we were analyzed by the MESMIS method a group of 25 environmental, social and economic sustainability indicators of a population of family farmers living in the Pedra Preta Watershed in Resende RJ. This proposal aimed to understand if there is sustainability in the property management model and to point the sustainability index for a community and properties that depend on dairy farming. Twenty-six properties were evaluated with the application of the indicators, which covered the socioeconomic and environmental dimensions, where the average sustainability index calculated was $67.8 \%$. According to the MESMIS classification, there are apparent weaknesses in wastewater treatment, lack of knowledge of agroecology and environmental education. Producers consulted were in favor of possible changes in management systems, but seem to lack technical support for the action.

KEYwORDs: Agroecology, diagnosis, indicators.

\section{Evaluación de la sostenibilidad del ganado lechero en la Cuenca Hidrográfica de Pedra Preta.}

RESUMEN - La determinación del índice de sostenibilidad agroecológica en las propiedades rurales es esencial para la dirección de las acciones de planificación y ejecución de políticas públicas de asistencia técnica y extensión rural, que promuevan 
mejoras en la calidad de vida de esta población. En este trabajo, fueron analizados por el método MESMIS en un grupo de 25 indicadores de sostenibilidad ambiental, social y económica de una población de agricultores familiares que viven en la cuenca de Pedra Preta en Resende RJ. Esta propuesta tuvo como objetivo comprender si hay sostenibilidad en el modelo de gestión de propiedades y señalar el índice de sostenibilidad para la comunidad y fincas que dependen de la producción lechera. Se evaluaron 26 fincas utilizando los indicadores, que fueron las variaciones socioeconómicas y ambientales, donde el índice de sostenibilidad promedio calculado fue del 67.8\%. Según la clasificación MESMIS, existen debilidades aparentes en el tratamiento de aguas residuales, falta de conocimiento de la agroecología y educación ambiental. Los productores consultados están a favor de posibles cambios en los sistemas de gestión, pero se ha observado que carecen de soporte técnico para la acción.

Palabras Clave: Agroecología, diagnóstico, indicadores.

\section{INTRODUÇÃO}

A agricultura de forma geral deve ter como objetivo a conservação e uso sustentável dos recursos, fazendo-se de grande importância à determinação da viabilidade e dos benefícios das práticas que atendam esses objetivos. Manter e melhorar a fertilidade e a estabilidade do solo, aumentar a diversificação das culturas e da renda, reduzir os usos de insumos químicos e água são ações de interesse em sistemas de transição agroecológica (Trabelsi et al. 2016; Cabanillas et al. 2017).

Mas, tratar do termo sustentabilidade é algo que vem sendo discutido por muitos autores desde a década de 80 (Veiga 2014), quando o termo ganhou destaque sendo adotado inclusive como jargão de marketing por muitas marcas e empresas, porém, não há nem entre os acadêmicos consenso sobre a definição da "sustentabilidade" principalmente pela sua multidisciplinariedade, ganhando muitos ramos filosóficos.

Por isso neste trabalho, os autores tratam o termo sustentabilidade no sentido de indicar o potencial de permanência espaço temporal de atividades agropecuárias, envolvendo aspectos de manutenção, recuperação, promoção socioeconômica, cultural e ambiental de unidades de produção agrícola, assim, relacionando que, quanto mais sustentável se apresentar uma propriedade, maior será seu índice de relacionamento com a agroecologia.

Nesse contexto torna-se fundamental a adoção dos princípios da agroecologia como norteadores do processo produtivo para conquista do desenvolvimento rural sustentável, conforme preconizam (Caporal e Ramos 2006). São definidos como agroecológicos aqueles sistemas que buscam a produção com menos uso de insumos químicos, utilização de recursos renováveis, manutenção da diversidade de espécies (Feiden 2005), isso implica na sustentabilidade ambiental em processos agrícolas, com maiores equidades social e econômica.

Oliveira e Salviano 2016, ao analisarem dados do Instituto Nacional de Colonização e Reforma Agrária, afirmam que entre os agricultores familiares, a bovinocultura de leite é uma das principais atividades desenvolvidas no Brasil, destacando que na região Sudeste esta prática está presente em cerca de $44 \%$ dos estabelecimentos rurais classificados como de economia familiar.

Em um estudo realizado no Sul de Minas Gerais, Bruhn et al. 2016 afirmam haver grande heterogeneidade no processo de produção, mas a maior parte do leite é produzida em pequenas fazendas que utilizam mão-de-obra familiar com tecnologias modernas, como inseminação artificial e ordenha mecânica. Sendo a produção leiteira de grande importância econômica por causa dos recursos humanos envolvidos nessa atividade, sua geração de renda e sua compatibilidade com a agricultura familiar (Dantas et al. 2016).

No estado do Rio de Janeiro, Souza et al. (2014) apontam a pecuária leiteira como característica em pequenas propriedades familiares e em Resende RJ, segundo o (IBGE 2014), este segmento movimentou cerca de 38 milhões de reais, com produção total de 32 milhões de litros de leite. 
Pensando em indicar o índice de sustentabilidade desses sistemas de produção, uma equipe multi-institucional chamada GIRA A.C. (Grupo Interdisciplinar de Tecnologia Rural Apropriada), no México, iniciou em 1995 o desenvolvimento do método MESMIS (Marco para la Evaluación de Sistemas de Manejo Incorporando Indicadores de Sostenibilidad), tendo como arcabouço o núcleo metodológico da Rede de Gestão de Recursos Naturais, (Speelman et al. 2007; Astier et al. 2012).

Essa metodologia permite analisar os índices sustentabilidade de propriedades agrícolas, proporcionando uma análise quantitativa rápida e de baixo custo de implementação, com grande eficiência e rapidez, e como concluem (Trabelsi et al. 2016).

Esta avaliação é fundamental para execução eficiente de diagnósticos capazes de apontar as fragilidades e potenciais dos sistemas de manejo adotados, pois, como salientam Dantas et al. (2016), estudos que caracterizam os sistemas produtivos são importantes para entender as prioridades e realidade das propriedades, sendo instrumento de apoio para elaboração de políticas públicas de assistência técnica e extensão rural (ATER).

Nesse contexto, o trabalho teve como objetivo a inovação e aplicação de métodos que permitiram indicar a situação econômica, social e ambiental de uma comunidade rural da Bacia Hidrográfica da Pedra Preta (BHPP), área rural do município de Resende RJ.

\section{Materiais e métodos}

\section{Área amostral}

A área avaliada se encontra no município de Resende no Estado do Rio de Janeiro (Figura 1). O recorte do local estudado conforma uma Bacia Hidrográfica (BH), conhecida como BH da Pedra Preta, com cerca de $80 \mathrm{Km}^{2}$. A BH possui $84,3 \mathrm{~km}$ de linhas de drenagem, sua foz se encontra em altitude de $424 \mathrm{~m}$ e seu ponto culminante a $1734 \mathrm{~m}$ resultando num gradiente altitudinal de $1310 \mathrm{~m}$.

Sua elevação é proeminente por se localizar na região da Serra da Mantiqueira Fluminense, onde são encontrados os pontos de maior altitude desse complexo montanhoso. Com relevo fortemente ondulado na sua parte mais plana e extremamente montanhoso na parte mais elevada apresentando declividades que impedem qualquer tipo de exploração agrícola.

Mesmo com relevo acidentado, a atividade característica da região é a pecuária de leite e corte, geralmente conduzida de maneira tradicional, com mão-de-obra principalmente familiar e de pouco tecnológica.

O clima é difícil de ser descrito devido ao seu largo gradiente de altitudes, mas de acordo com a classificação de (Köppen 1948) pode ser definido como Cfa (subtropical, com verão quente), que abrange as regiões sul de Minas Gerais, sudeste dos estados do Rio de Janeiro e São Paulo. 
Figura 1. Localização da área de estudo em relação ao território nacional.

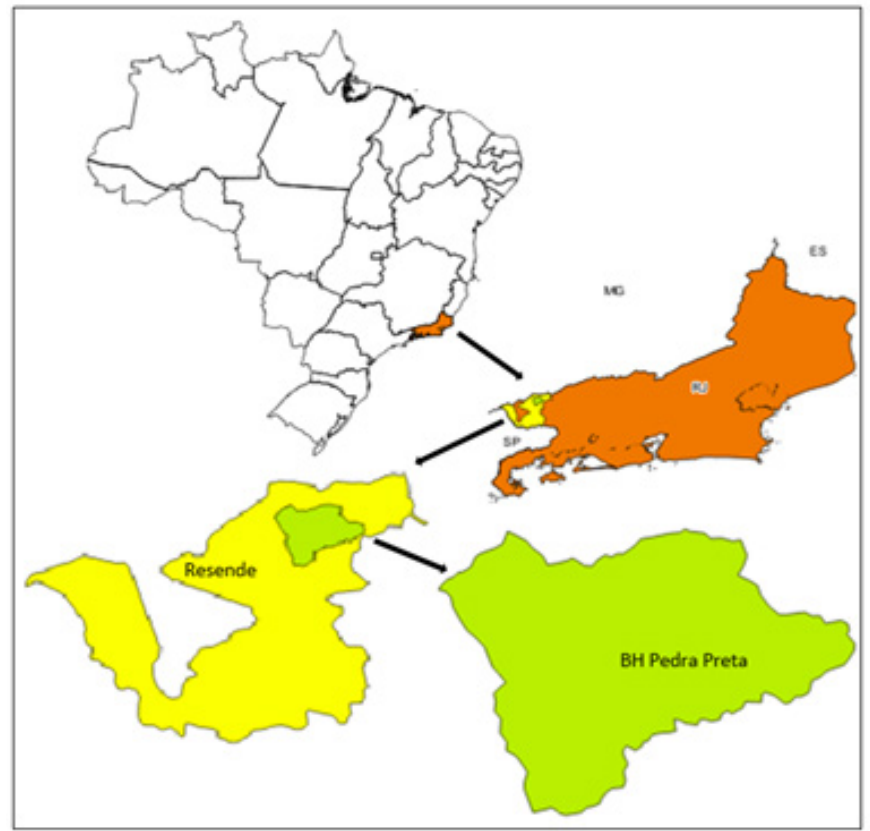

Amostragem e critérios de inclusão

A amostragem foi conduzida de novembro de 2016 a fevereiro de 2017. Todos os participantes concordaram espontaneamente em participar da pesquisa e tomaram conhecimento do Termo de Consentimento Livre e Esclarecido (TCLE) aprovado pelo Comitê de Ética e Pesquisa (CEP) da Universidade Federal de São Carlos (Parecer número 1.824.674).

Os entrevistados se enquadraram nos seguintes critérios de inclusão: concordaram em participar voluntariamente, eram produtores rurais, residiam na propriedade ou dentro dos limites da $\mathrm{BH}$, obtinham do manejo da terra sua principal fonte de renda e sustento.

Para realização das entrevistas e verificação dos proprietários que atendiam aos critérios de corte desta pesquisa, todas as 53 propriedades rurais situadas dentro do limite geográfico da $\mathrm{BH}$ foram visitadas pelo pesquisador.

As questões foram elaboradas abordando as três bases fundamentais da agroecologia: ambiental (nove questões), social e econômica (oito questões cada). Os indicadores propostos no formulário foram elaborados com base na bibliografia (Guimarães et al. 2015; Gallo et al. 2016; Barbosa et al. 2017; Cabanillas et al. 2017), e no conhecimento dos autores deste trabalho sobre as características da área de estudo.

Foram analisados através dos indicadores situações como: manejo do solo e dos animais; sistemas de pastoreio adotados; dependência de insumos; aplicação de agrotóxicos e medicamentos; convívio com na comunidade; acesso a informação; escolaridade; projetos de ATER; beneficiamento de produtos; comercialização; investimentos entre outros.

\section{Análises}

Os indicadores aplicados foram classificados de acordo com a metodologia MESMIS, onde cada indicador recebeu uma pontuação de um a três, sendo que (um) representa situação indesejável, (dois) situação regular e (três) condição desejável com práticas voltadas à agroecologia (Speelman et al. 2007; Guimarães et al. 2015; Silva et al. 2015; Gallo et al. 2016; Barbosa et al. 2017). 
As propriedades foram classificadas em cinco categorias de vulnerabilidade a partir do somatório da pontuação dos indicadores, onde para as dimensões social econômica que poderiam obter pontuação mínima de 8 e a máxima 24. Portanto de 8 a 11 pontos muito frágil, 12 a 14 frágil, 15 a 17 regular, 18 a 20 forte e $>20$ muito forte. Para a dimensão ambiental com pontuação mínima de 9 e máxima de 27, então de 9 a 12 pontos muito frágil, 13 a 16 frágil, 17 a 20 regular, 21 a 24 forte e > 24 muito forte (pontuações adaptadas de Rempel et al. 2012; Guimarães et al. 2015; Gallo et al. 2016).

Para avaliar o desempenho dos indicadores aplicados, foi extraída a média aritmética de cada um deles a fim de apontar o índice de vulnerabilidade em cada item.

\section{Propriedades}

$\mathrm{Na}$ avaliação do índice sustentabilidade das propriedades foram utilizadas as seguintes expressões:
$\boldsymbol{I S A E}=\sum I N / N Q$
e $\quad \boldsymbol{I T}=I S A E / 3 * 100$

Onde:

ISAE= Índice de sustentabilidade socioambiental e econômica (média MESMIS)

$\mathrm{IN}=$ soma dos indicadores

$\mathrm{NQ}=$ número de questões

IT $=$ índice de sustentabilidade

Dessa forma foi possível verificar para cada propriedade seu índice de sustentabilidade em uma escala de 0 a 100, sendo que zero expressa situação de insustentabilidade e manejo convencional e 100 expressa situação oposta, totalmente sustentável e com manejo agroecológico.

\section{Resultados E Discussões}

\section{Indicadores de sustentabilidade, pontos frágeis e fortes}

Os indicadores ambientais avaliados apresentaram variação entre as médias de 1,26 pontos. Os mais frágeis se referem aos processos de formação de pastagens e tratamento de efluentes que apresentaram respectivamente média de 1,5 e 1,65 pontos (Figura 2, quadro A), sendo que na formação dos pastos em geral os processos mais citados foram a utilização de aração profunda e introdução de braquiaria, situação também verificada por Barbosa et al. (2017) que relacionam pontos frágeis nas áreas de pastagem ao fato da área ser uma área de pastagem, basicamente composta por uma espécie, Bracbiaria decumbens, ocasionando a redução da diversidade, entre outros fatores com implicações negativas para a sustentabilidade ambiental.

Quanto ao tratamento de efluentes, este se encontra ausente na maioria das propriedades, sendo lançado in natura no solo ou cursos d'água.

Vieira et al. (2015) destacam a gravidade da falta de tratamento de resíduos e efluentes onde estes podem ser considerados uma questão socioambiental, por estar relacionado à saúde pública, tem repercussões sobre a preservação e/ou conservação dos recursos naturais, principalmente, no que tange aos mananciais hídricos. 
O indicador com melhor desempenho foi o de qualidade e disponibilidade de recursos hídricos, com 2,76 pontos, isso se deve ao fato de que quase a totalidade dos produtores terem como fonte de abastecimento pequenas nascentes no interior das propriedades, não utilizando água do rio principal da $\mathrm{BH}$.

Os indicadores sociais apresentaram variação de 1,07 pontos. O indicador com a menor média foi o do posicionamento pessoal dos agricultores em relação a transição agroecoecológica com média de 1,73 pontos, pois na maioria das vezes afirmaram desconhecer o termo e a maior média foi 2,8 pontos em relação ao indicador de origem e cultura da família, compreendendo que quase todos são residentes locais e com histórico familiar camponês (Figura 2, quadro B). Na dimensão econômica foi encontrada a maior variação dentre as médias dos indicadores (1,39 pontos). A menor média encontrada foi 1,03 pontos referente a alimentação do gado, essa pontuação é muito baixa e considerada como indesejável pela escala MESMIS, isso ocorreu devido a maioria dos produtores adquirirem de forma individual e através de atravessadores os complementos alimentares e medicamentos para os rebanhos, além de muitos não contarem com capineiras, canaviais ou silagem em suas propriedades, ocosionando aumento significativo no custo de produção.

Figura 2. Desempenho dos A) indicadores ambientais, B) sociais e C) econômicos avaliados pelo método MESMIS (escala de 1- indesejável a 3 - desejável) das propriedades analisadas na BHPP, Resende, RJ.
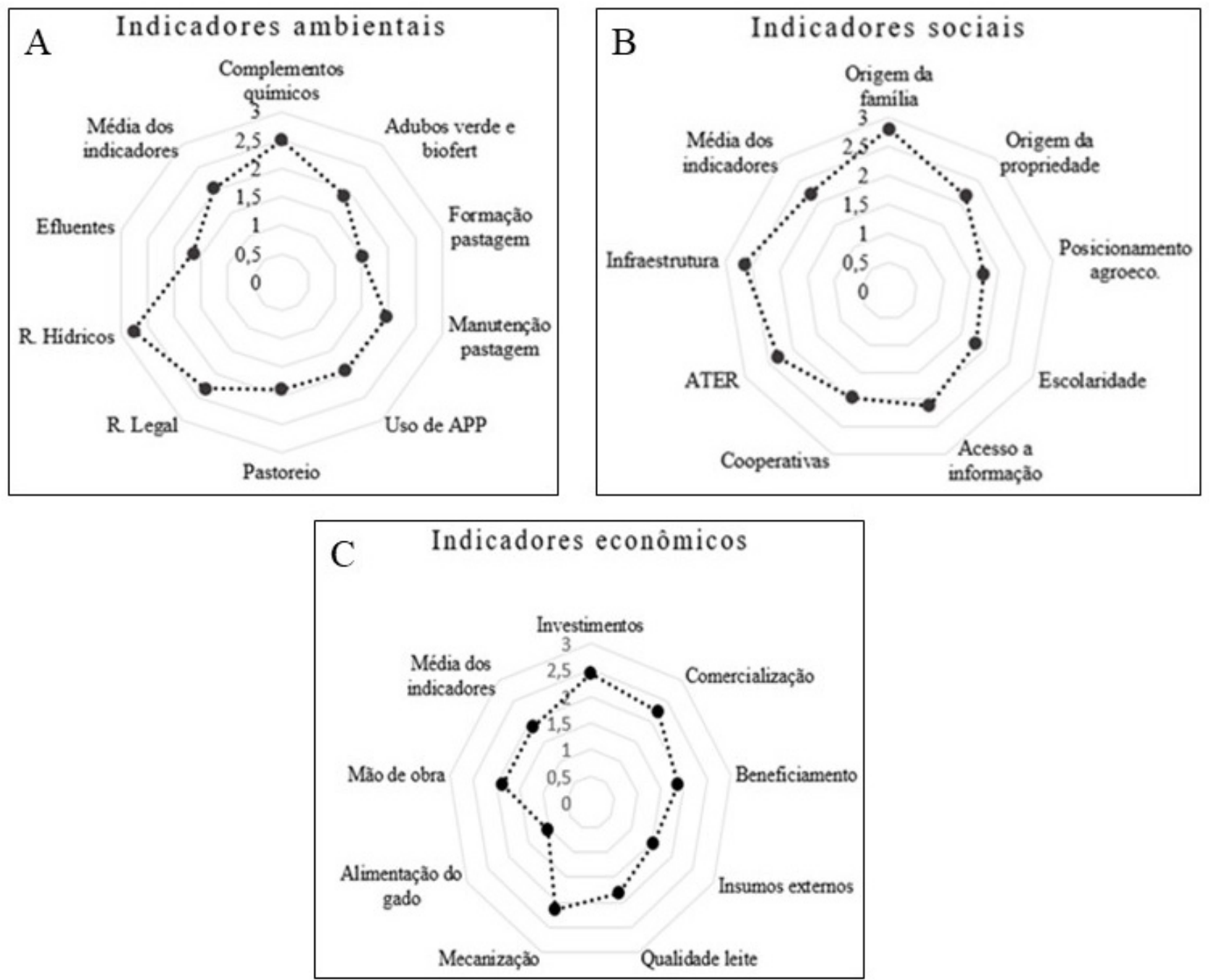
A maior média para dimensão econômica foi 2,42 no indicador de investimentos e atualização dos sistemas, em que a maior parte dos proprietários possuem sistemas mecânicos de ordena e armazenagem dos produtos (Figura 2, quadro C).

A tecnificação do sistema de ordenha e armazenamente reflete que os proprietários veem atualizando constantemente seus sistemas de trabalho, e tal situação se assemelha ao estudo realizado por Bruhn et al. (2016) na região Sul do Estado de Minas Gerais no qual relatam heterogeneidade no processo de produção em relação a dimensão das propriedades, mas a maior parte do leite é produzidao em pequenas fazendas que utilizam mão-deobra familiar com tecnologias modernas, como inseminação artificial e ordenha mecânica.

Esta avaliação permitiu a identificação dos indicadores de sustentabilidade com maior fragilidade, pois, aqui foi avaliado a média de cada indicador para a comunidade. Os pontos frágeis, se apresentam como elementos chave num diagnóstico uma vez que, esclarecem as limitações ou potencialidades da manutenção do sistema ao longo do tempo (Deponti et al. 2002) podendo ser considerados como pontos de partida para rearranjos no modelo de manejo adotado, sendo possível atingir o redesenho integral do sistema de produção.

A metodologia MESMIS foi muito útil nesta situação pois, permitiu a adaptação para diferentes áreas e atividades agrícolas como citado nos trabalhos de (López-Riadura et al. 2002; Sarandón e Flores 2009; Guimarães et al. 2015; Gallo et al. 2016), ainda destacam que a metodologia permite derivação, medição e monitoramentos de indicadores de sustentabilidade como parte de uma abordagem sistêmica e participativa, conferindo-lhe características de um instrumento de avaliação flexível e adaptável a distintas situações de estudo.

Gallo et al. (2016), afirmam que esses pontos são norteadores de ações mitigadoras, que vizem a busca equilíbrio pleno do sitema de produção. Identificar esses pontos contribui positivamente na elaboração de propostas e planos de ação em ATER para as famílias camponesas na busca de sistemas de produção estável/dinâmico com características de se abalar minimamente ante processos de disturbio, demosntrando a sustentabilidade do sistema e fortalecendo o processo de aproximação da agroecologia.

As informações levantadas apontam claramente para as demandas de extensão rural com práticas de educação ambiental nessa comunidade, uma vez que foi observado o desconhecimento dos termos "Agroecologia" e “ATER”, os quais são elementos essenciais no processo de transição agroecológica e sustentabilidade.

Como destacam Caporal e Ramos (2006) é importante que se considere o atendimento de demandas pontuais dos produtores, podendo o extensionista atuar de forma planejada regionalmente de acordo com as necessidades da comunidade. A avaliação de sustentabilidade, no entanto, indica a direção, a prioridade das mudanças e propostas para contribuir com um desenvolvimento sustentável baseados nos agroecossistemas (Kemerich et al. 2013).

Analisando as informações mencionadas pelos produtores da ocorrência de pragas nas pastagens como mexerico (Leandra aurea), presença de parasitas nos animais (principalmente, larvas de Dermatobia hominis e carrapatos) e alta contagem de células somáticas (CCS) causadoras de mamites, pode-se indicar que na mudança nos sistemas atuais de manejo convencional para o manejo agroecológico dos rebanhos e pastagens, esses pontos frágeis devem ser mitigados.

Pois, Silva et al. (2014) estudando rebanhos leiteiros criados sob sistemas orgânicos, verificaram que esses tiveram menor mortalidade de prole, menor incidência de mastite, menores taxas de abortamento, bem como contagem mais baixa de carrapatos e piolhos. Souza et al. (2014), ao analisar o desempenho econômico e produtivo de propriedades atuantes na pecuária leiteira no Estado do Rio de Janeiro, afirmam ter obtido rendimento financeiro $30 \%$ superior nos sistemas agroecológicos quando comparados aos convencionais.

Ambos os autores concluem que o manejo orgânico ou agroecológico de rebanhos e pastagens ocasionam maior rendimento econômico pelo aumento da qualidade do leite e ainda ganhos ambientais com a redução de pragas como Leandra aurea que de acordo com Robinson et al. (2015) é relacionada negativamente com a biomassa e diversidade de árvores onde são muito dominantes. 
Quanto às questões econômicas, os indicadores apontam fragilidade aparente na ausência de cooperação nos processos de compra de insumos entre os proprietários, sendo que a situação apresentada mostra um elevado custo na aquisição desses produtos que são negociados individualmente pelos proprietários com atravessadores.

Indicando que há desunião entre os produtores ao se tratar de relações econômicas, pois deveriam propor metodologias de aquisição e venda de produtos de formas conjunta, pois conforme Santos et al. (2016) essas entidades só funcionam com ação de indivíduos que buscam de forma solidária a obtenção de resultados econômicos que permitam a melhoria do nível de vida de cada um e de todos, e propicia desenvolvimento não só para a classe ruralista, mas para a comunidade em geral.

A organização de cooperativas ou de associação de produtores rurais tem o poder de envolver as comunidades de forma concreta, e serve de propulsor para melhoria da qualidade da produção e a viabilidade econômica da atividade leiteira. A soberania tecnológica é um dos principais objetivos da transição para a produção agroecológica (Cabanillas et al. 2017).

\section{Vulnerabilidade socioeconômica e ambiental das propriedades}

Os resultados obtidos dos indicadores de sustentabilidade ambiental apontam 7 propriedades em situação frágil, 12 em situação regular e 7 propriedades fortes.

Com os indicadores sociais, foi possível identificar 3 propriedades em situação frágil, 10 propriedades regulares, 9 fortes e 4 muito forte em relação a sustentabilidade das relações sociais das famílias (Quadro 1).

Nos indicadores de sustentabilidade econômica foram identificadas 3 propriedades em situação muito frágil, 7 em situação frágil, 14 em regular e 2 propriedades como fortes em relação a sustentabilidade econômica dos entrevistados (Quadro 1).

As propriedades 3, 9, 11 e 12 relacionadas no (Quadro 1) apresentaram os melhores desempenhos quanto a classificação de fragilidade, o que indica que em caso de proposição de ações de ATER essas propriedades podem ser relatadas como exemplos regionais de manejo. As propriedades 3 e 10 apresentaram os piores desempenhos na classificação de vulnerabilidade. A média dos indicadores analisados para a BH obtiveram classificação regular para as três dimensões analisadas.

Quadro 1. Classificação das propriedades em relação ao somatório dos indicadores socioambiental e econômico, conforme as $\mathbf{5}$ classes propostas na metodologia.

\begin{tabular}{|c|c|c|c|c|c|c|}
\hline Propriedade & $\begin{array}{c}\text { Indicadores } \\
\text { ambientais }\end{array}$ & Classificação & $\begin{array}{c}\text { Indicadores } \\
\text { sociais }\end{array}$ & Classificação & $\begin{array}{c}\text { Indicadores } \\
\text { econômicos }\end{array}$ & Classificação \\
\hline PP_001 & 15 & Frágil & 13 & Frágil & 16 & Regular \\
\hline PP_002 & 15 & Frágil & 18 & Forte & 14 & Frágil \\
\hline PP_003 & 17 & Regular & 16 & Regular & 10 & Muito frágil \\
\hline PP_004 & 19 & Regular & 19 & Forte & 17 & Regular \\
\hline PP_005 & 16 & Frágil & 19 & Forte & 14 & Frágil \\
\hline PP_006 & 14 & Frágil & 18 & Forte & 14 & Frágil \\
\hline PP_007 & 14 & Frágil & 16 & Regular & 18 & Forte \\
\hline PP_008 & 17 & Regular & 22 & Muito forte & 17 & Regular \\
\hline PP_009 & 21 & Forte & 21 & Muito forte & 17 & Regular \\
\hline PP_010 & 16 & Frágil & 14 & Frágil & 13 & Frágil \\
\hline PP_011 & 20 & Regular & 22 & Muito forte & 16 & Regular \\
\hline PP_012 & 21 & Forte & 20 & Forte & 17 & Regular \\
\hline
\end{tabular}




\begin{tabular}{|c|c|c|c|c|c|c|}
\hline PP_013 & 21 & Forte & 19 & Forte & 11 & Muito frágil \\
\hline PP_014 & 20 & Regular & 13 & Frágil & 14 & Frágil \\
\hline PP_015 & 18 & Regular & 16 & Regular & 17 & Regular \\
\hline PP_016 & 13 & Frágil & 17 & Regular & 17 & Regular \\
\hline PP_017 & 20 & Regular & 17 & Regular & 16 & Regular \\
\hline PP_018 & 22 & Forte & 15 & Regular & 15 & Regular \\
\hline PP_019 & 20 & Regular & 17 & Regular & 16 & Regular \\
\hline PP_020 & 18 & Regular & 17 & Regular & 14 & Frágil \\
\hline PP_021 & 21 & Forte & 16 & Regular & 15 & Regular \\
\hline PP_022 & 21 & Forte & 15 & Regular & 17 & Forte \\
\hline PP_023 & 22 & Forte & 19 & Forte & 10 & Muito frágil \\
\hline PP_024 & 19 & Regular & 18 & Forte & 15 & Regular \\
\hline PP_025 & 18 & Regular & 18 & Forte & 16 & Regular \\
\hline PP_026 & 20 & Regular & 21 & Muito forte & 13 & Frágil \\
\hline Média & $\mathbf{1 8}$ & Regular & 17 & Regular & 15 & Regular \\
\hline
\end{tabular}

A realização de diagnóstico é de grande valia no entendimento das necessidades dos produtores da região, pois a partir dos resultados, permite-se propor ações corretivas e mitigatórias de pontos críticos e realizar um acompanhamento dos mesmos ao longo do tempo (Sarandón e Flores, 2009). Pois como comenta (Souza 2009) técnicas de diagnóstico e planejamento devem focar no processo de aquisição de dados e informações, e que neste processo se permita um ato de formação e discussão de políticas na comunidade sendo ela a principal geradora e beneficiada desses dados.

Roboredo et al. (2015) afirmam esses diagnósticos permitem a implementação de políticas públicas e é indispensável, por exemplo, em programa de consevação de recursos e caracteríticas naturais nas microbacias hidrográficas. Nesse caso é possível observar se existem aspectos emergenciais a serrem tratados e em quais propriedades devem ser focados os esforços no sentido da promoção de ATER.

Numa avaliação de sustetabilidade ambiental no meio rural, (Rempel et al. (2012) comentam sobre a importância de se avaliar os índices de todas as propriedades de forma conjunta, pois permite a identificação das ações e práticas que se expõe a riscos de qualidade ambiental.

Como trata-se não só da sustentabilidade, mas também da avaliação de sistemas agroecológicos de produção, Gallo et al. (2016) escreve sobre a necessidade da avaliação das dimensões social, ambiental e econômica, que estão intimamente envolvidas no âmbito da produção agrícola sustentável, pois norteiam o uso e manejo de recursos humanos, naturais e financeiros.

\section{Índice de sustentabilidade}

$\mathrm{Na}$ avaliação do índice de sustentabilidade das propriedades foram encontrados valores percentuais variando de $57,3 \%$ a 78,7\% referentes às propriedades 3, 10 e 9 respectivamnte, e para a comunidade o índice calculado foi de $67,8 \%$ (Figura 3). 
Figura 3. Indice de sustentabilidade calculado para cada propriedade na BH Pedra Preta pela expressão $I T=$ $I S A E / 3$ * 100, onde: IG lê-se índice geral que se refere à média obtida para a comunidade.

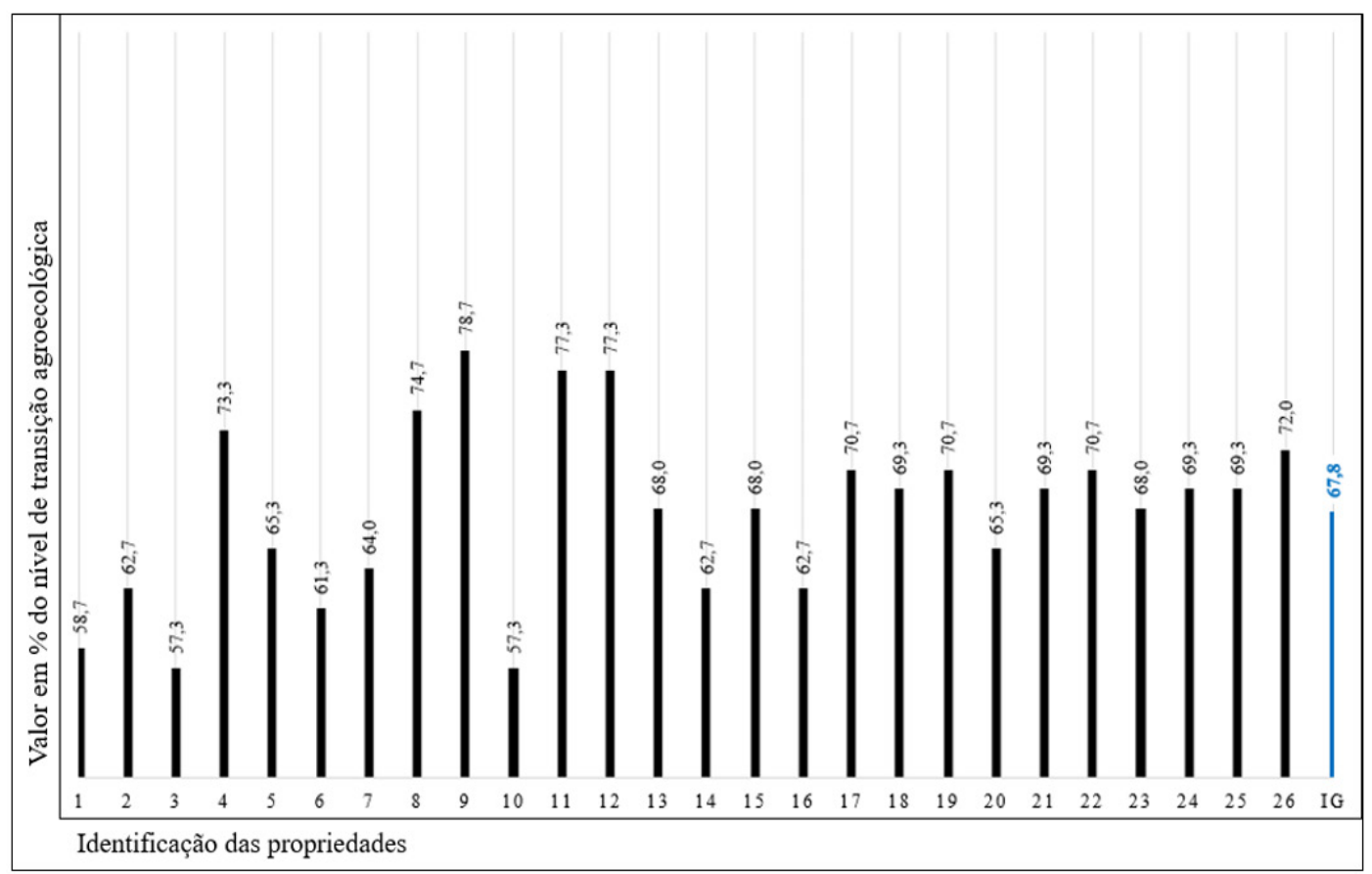

Com os resultados do índice de sustentabilidade proposto, observados os indicadores e propriedades de maior fragilidade, pode-se propor de forma assertiva medidas mitigatórias, em prol da melhoria de todos os aspectos sociais econômicos e ambientais da $\mathrm{BH}$.

Uma das melhorias seria com a mudança para sistemas agroecológicos de manejo. Oliveira et al. (2015) demonstrou que 19 dos 25 indicadores analisados por eles apresentaram melhoria significativa e conclui que os resultados apontam que a transição do convencional para o manejo orgânico se mostra eficiente pelos índices obtidos dos impactos socioambientais e ecológicos. Como nas palavras de Bonaudo et al. (2014), os desafios de alcançar a sustentabilidade requerem progressos simultâneos na produção e no desempenho ambiental.

Fazendo o uso das abordagens alternativas nos sistemas de produção proposto pelas ideologias agroecológicas que conforme discorre Altieri (2002) baseiam-se na utilização dos recursos disponíveis localmente tanto quanto possível, embora não rejeitem totalmente o uso de insumos externos.

Contudo, os agricultores não devem tentar se beneficiar dos recursos/insumos que não estão disponíveis, acessíveis ou adequados às suas condições.

\section{Conclusão}

Entre os indicadores avaliados, o diagnóstico mostra que a comunidade apresenta alguns pontos de fragilidade na dimensão econômica como deficiência na cooperação financeira entre os produtores, na ambiental apresenta problemas quanto aos sistemas de tratamento de efluentes e desperdícios de energia por meio do descarte inadequado de estercos que se tornam um problema pela falta de manejo, uma vez que, podem ser convertidos em fertilizantes para a propriedade ou comercializados. Na dimensão social o quadro é mais estável, apesar do desconhecimento da própria agroecologia e pouca noção de educação ambiental.

De modo geral, as propriedades apresentam bom índice em relação à sustentabilidade e agroecologia e os produtores abordados se mostraram favoráveis às mudanças em busca da melhoria dos sistemas e consequente redução dos gastos e desperdícios. 
O método de indicação de sustentabilidade numa escala de 0 a 100, foi proposto a partir dos resultados obtidos com a aplicação dos índices MESMIS, e mostrou-se muito útil, permitindo visualização da tendência das propriedades em relação à agroecologia e conservação dos recursos naturais.

Cabe ressaltar que o índice de sustentabilidade identificado nas propriedades poderá ser modificado com a ampliação do estudo, e recomenda-se que seja feito, abordando áreas mais técnicas como sanidade animal, avaliação físico química do solo e água, produtividade por animal.

\section{Agradecimentos}

Agradecemos imensamente aos proprietários e residentes da BH Pedra Preta pelo gentil acolhimento e disponibilidade quando solicitados a participar desta pesquisa. A Universidade Federal de São Carlos, Centro de Ciências Agrárias-Araras, onde este trabalho pôde ser redigido. A CAPES (Coordenação de Aperfeiçoamento Pessoal de Nível Superior) pela concessão da bolsa de estudos.

\section{REFERÊNCIAS}

Abbona EA, Sarandón SJ, Marasas ME and Astier, M. 2007. Ecological sustainability evaluation of traditional management in different vineyard systems in Berisso, Argentina. Agriculture, Ecosystems \& Environment, v. 119, n. 3, p. 335-345.

Altieri MA. 2002. Agroecology: the science of natural resource management for poor farmers in marginal environments. Agriculture, ecosystems \& environment, v. 93, n. 1, p. 1-24.

Astier M, García-Barrios L, Galván-Miyoshi Y, González-Esquivel C, and Masera O. 2012. Assessing the sustainability of small farmer natural resource management systems. A critical analysis of the MESMIS program (1995-2010). Ecology and society, v. 17, n. 3.

Barbosa, MM, Reis JD, Giunti, OD and Silva AV. 2017. Indicadores de sustentabilidade em duas áreas distintas, em Caldas/ MG, através da metodologia MESMIS. Holos Environment, v. 17, n. 1, p. 1-14.

Bonaudo T, Bendahan AB, Sabatier R, Ryschawy J, Bellon S, Leger F, and Tichit M. 2014. Agroecological principles for the redesign of integrated crop-livestock systems. European Journal of Agronomy, v. 57, p. 43-51.

Bruhn FRP, Daher DO, Rocha CMBM, Barbieri JM, Lucci J R, and Guimarães AM. 2016. Zootechnical profile of the dairy farms in southern Minas Gerais State, Brazil. Archivos Latinoamericanos de Producción Animal, v. 24, n. 3.

Cabanillas C, Tablada M, Ferreyra L, Pérez A, and Sucani G. 2017. Sustainable management strategies focused on native bioinputs in Amaranthus cruentus L. in agro-ecological farms in transition.Journal of Cleaner Production, v. 142, p. 343-350.

Caporal FR e Ramos LF. 2006. Da extensão rural convencional à extensão rural para o desenvolvimento sustentável: enfrentar desafios para romper a inércia. Belém: UFPA. 26 p.

Dantas VV, Oaigen RP, Santos MAS, Godoy BS, Silva F, Corrêa RP and Marques CSS. 2016. Characteristics of cattle breeders and dairy production in the southeastern and northeastern mesoregions of Pará state, Brazil. Semina: Ciências Agrárias, v. 37 , n. 3 , p. $1475-1488$. 
Deponti CM, Eckert C e Azambuja, JLB. 2002. Estratégia para construção de indicadores para avaliação da sustentabilidade e monitoramento de sistemas. Agroecologia e desenvolvimento rural sustentável, v. 3, n. 4, p. 44-52.

Flores CC and Sarandón SJ. 2015. Evaluation of the sustainability of a agroecological transition process in familiar horticultural systems of La Plata, Buenos Aires, Argentina. Revista de la Facultad de Agronomía (La Plata), 114(3), 52-66.

Feiden A. 2005. Agroecologia: Introdução e conceitos. In: Agroecologia: Princípios e técnicas para uma agricultura orgânica sustentável. $1^{a}$ ed. Brasília: Embrapa Informação Tecnológica, p. 51 - 70.

Gallo AS, Guimarães NF, Cunha C, Santos RDP e Carvalho EM. 2016. Indicadores da sustentabilidade de uma propriedade rural de base familiar no estado de Mato Grosso do Sul. Revista Verde de Agroecologia e Desenvolvimento Sustentável, v. 11, n. 3, p. 104-114.

Guimarães NF, Gallo AS, Santos CC, Morinigo KPG, Bentos AB e Carvalho, E. M. 2015. Avaliação da sustentabilidade de um agroecossistema pelo método MESMIS. Scientia Plena, v. 11, n. 5.

IBGE. Disponível em: http:/ $/$ www.cidades.ibge.gov.br/xtras/temas.php?lang=\&codmun=330420\&idtema=147\&search $=\mathrm{r}$ io-de-janeiro | resende | pecuaria-2014. Acesso em: 14 de set. 2015.

Kemerich PDC, Martins SR, Kobiyama M, Buriol GA, Borba WFe Ritter LG. 2013. Avaliação da sustentabilidade ambiental em bacias hidrográficas mediante a aplicação do modelo PER. Electronic Journal of Management, Education and Environmental Technology (REGET), v. 10, n. 10, p. 2140-2150.

Köppen, W. Climatologia:con un estudio de los climas de la tierra. Fondo de Cultura Econômica. México. 479p. 1948.

López-Riadura S, Masera O and Astier M. 2002. Evaluating the sustainability of complex sócio-environmental systems, the Mesmis framework. Ecological Indicators, v. 2, p. 135-148.

Oliveira AF, Salviano FA. 2016. Bovinocultura de leite em assentamentos de Mato Grosso baseado no modelo Mesmis. Revista Meio Ambiente e Sustentabilidade, v. 10, n. 5, p. 128-150.

Oliveira ER, Muniz EB, Soares JPG, Carbonari VMS, Carbonari OS, Gabriel AMG e Gandra JR. 2015. Impactos Ecológicos e Socioambientais da Transição Agroecológica para produção orgânica de Leite em Sidrolândia-MS. Cadernos de Agroecologia, v. 9, n. 4.

Rempel C, Eckhardt RR, Jasper A, Schultz G, Hilgert ÍH e Barden JE. 2012. Proposta metodológica de avaliação da sustentabilidade ambiental de propriedades produtoras de leite. Tecno-Lógica, v. 16, n. 1, p. 48-55.

Robinson SJB, van den Berg E, Meirelles GS end Ostle N. 2015. Factors influencing early secondary succession and ecosystem carbon stocks in Brazilian Atlantic Forest. Biodiversity and conservation, v. 24, n. 9, p. 2273-2291.

Roboredo D, Bergamasco SMPP e Bleich ME. 2016. Aggregate index of socialcenvironmental sustainability to evaluate the socialcenvironmental quality in a watershed in the southern amazon. Ecological Indicators, 63: 337 - 345.

Santos VF, Guimarães LHWS, Nobrega TC, e Moreira FG. 2016. A comercialização de hortaliças na agricultura familiar: uma análise no assentamento Santa Olga, Nova Andradina/MS. Revista Desenvolvimento Social, v. 1, n. 16. 
Sarandón SJ y Flores CC. 2009. Evaluación de la sustentabilidad en agroecosistemas: una propuesta metodológica. Agroecología, v. 4, p. 19-28.

Silva JB, Fagundes GM, Soares JP, Fonseca AH and Muir JP. 2014. A comparative study of production performance and animal health practices in organic and conventional dairy systems. Tropical animal health and production, v. 46, n. 7, p. 1287-1295.

Silva NC; Santos CFB e Nascimento AD. 2015. Diagnóstico de Indicadores de Sustentabilidade em Agroecossistemas no Município de Glória de Dourados-MS. Cadernos de Agroecologia, v. 9, n. 4.

Souza MMO. 2009. A utilização de metodologias de diagnóstico e planejamento participativo em assentamentos rurais: o diagnóstico rural/rápido participativo (DRP). Em Extensão. Uberlândia v. 8, n. 1, p. 34 - 47.

Souza SO, Castagna AA, Ferreira JM, Valentini L, Oliveira LAA e Santos JGC. 2014. Produção de leite em sistema de manejo agroecológico de pastagem (Microbacia Valão do Papagaio - Itaocara - RJ). Gerenciamento Integrado de Agroecossistemas em Microbacias Hidrográficas do Norte e Noroeste Fluminense.

Speelman EM, López-Ridaura S, Colomer NA, Astier M and Masera OR. 2007. Ten years of sustainability evaluation using the MESMIS framework: Lessons learned from its application in 28 Latin American case studies. The International Journal of Sustainable Development \& World Ecology, v. 14, n. 4, p. 345-361.

Trabelsi M, Mandart E, Le Grusse P, and Bord JP. 2016. How to measure the agroecological performance of farming in order to assist with the transition process. Environmental Science and Pollution Research, v. 23, n.1, p. 139-156.

Veiga, JED. 2014. O âmago da sustentabilidade. Estudos avançados, v.28, n. 82, p. 7-23.

Vieira LR; Vieira, LR; Vestena S. 2015. A questão do saneamento no espaço rural: uma abordagem ambiental em três localidades rurais no município de Nova Palma, RS. Electronic Journal of Management, Education and Environmental Technology (REGET), v. 19, n. 1, p. 38-50. 